Preprint typeset in JHEP style - HYPER VERSION

\title{
Dark matter annihilation and non-thermal Sunyaev-Zel'dovich effect: II. dwarf spheroidal galaxy
}

\author{
Feng Huang ${ }^{a, b}$, Xuelei Chen ${ }^{a, c}$, Qiang Yuan ${ }^{d, e}$, Xiaojun $\mathbf{B i}^{d, c}$ \\ ${ }^{a}$ National Astronomical Observatories, Chinese Academy of Sciences, Beijing 100012, \\ China \\ ${ }^{b}$ Department of Physics and Institute of Theoretical Physics and Astrophysics, Xiamen \\ University, Xiamen, Fujian 361005, China \\ ${ }^{c}$ Center of High Energy Physics, Peking University, Beijing, 100871, China \\ ${ }^{d}$ Key Laboratory of Particle Astrophysics, Institute of High Energy Physics, Chinese \\ Academy of Sciences, Beijing 100049,China \\ ${ }^{e}$ Graduate University of Chinese Academy of Sciences, Beijing 100049, China
}

\begin{abstract}
We calculate the CMB temperature distortion due to the energetic electrons and positrons produced by dark matter (DM) annihilation (Sunyaev-Zel'dovich effect, $\mathrm{SZ}_{\mathrm{DM}}$ ) in dwarf spheroidal galaxies (dSphs). In the calculation we have included two important effects which were previously ignored. First we show that the $e^{ \pm}$with energy less than $\sim \mathrm{GeV}$, which were neglected in previous calculation, could contribute a significant fraction of the total signal. Secondly we also consider the full effects of diffusion loss, which could significantly reduce the density of $e^{ \pm}$at the center of cuspy halos. For neutralinos, we find that detecting such kind of SZ effect is beyond the capability of the current or even the next generation experiments, which is consistent with the quantitative description made by S. Colafrancesco in [1]. In the case of light dark matter (LDM) the signal is much larger, but even in this case it is only marginally detectable with the next generation of experiment such as ALMA. We conclude that similar to the case of galaxy clusters, in the dwarf galaxies the $\mathrm{SZ}_{\mathrm{DM}}$ effect is not a strong probe of $\mathrm{DM}$ annihilations.
\end{abstract}

KEywords: dark matter theory, Sunyaev-Zel'dovich effect, dwarf spheroidal galaxies. 


\section{Contents}

1. Introduction 1

2. Method of Calculation 2

2.1 The nonthermal SZ Effect 2

2.2 dark matter distribution

$2.3 e^{ \pm}$production from DM annihilation

$2.4 e^{ \pm}$propagation in dwarf galaxies

3. Results 10

3.1 Distribution of electrons 10

3.2 The $\mathrm{SZ}_{\mathrm{DM}}$ signal 11

4. Conclusion 16

\section{Introduction}

The physical nature of dark matter (DM) is a great unsolved problem in modern cosmology. A major observational approach to this problem is to look for possible signatures of DM annihilations. In many theoretical models, the DM particles could annihilate and produce $\gamma$-ray photons and other energetic particles such as electrons and positrons. This is the case, for example, for the supersymmetric DM model [2], and the light dark matter (LDM) model [3]. For some early investigations on this subject, see e.g. [ [4, 同, 6, 7, 8].

Here we consider the energetic electrons and positrons produced by such annihilations. One possible way to reveal or constrain the presence of such energetic electrons and positrons is to look for the Sunyaev-Zel'dovich (SZ) effect induced by them $\left(\mathrm{SZ}_{\mathrm{DM}}\right)$. Energetic electrons or positrons could scatter with cosmic microwave background (CMB) photons, producing a distortion in the CMB spectrum. In clusters of galaxies, the virial temperature is sufficiently high that this effect could be produced by thermal electrons, which is the so called thermal SZ effect [9]. The bulk movement of electrons with respect to the CMB also cause a similar effect, which is dubbed the kinetic SZ effect [10]. In galaxies, however, the virial temperature is too low for the thermal electrons to produce significant thermal SZ effect, and the number of free electrons is also too low to produce significant kinetic SZ effect. However, non-thermal energetic electrons and positrons do exist such as cosmic rays in galaxies, which may produce an additional non-thermal SZ effect. In particular, DM annihilation may supply some of such energetic particles. However, DM annihilation is not necessarily the only source of cosmic ray particles. A number of "normal" astrophysical sites, such as supernova remnants, AGN jets, pulsar winds etc., may 
also be partially responsible for cosmic rays. This is why the dwarf spheroidal galaxies (dSphs) may be of particular interest to us, as they are DM dominated and also considered to be very inactive, hence observations are less affected by background originating from normal astrophysical processes such as star formation and AGN activity.

The SZ effect induced by a non-thermal distribution of charged particles were studied in [11, 12], and then applied to clusters of galaxies [13, 14, 15] and dSphs, where the Draco dwarf was taken as an prime example [16]. These studies suggest that the DM-induced SZ effect could be used to search for signatures of DM annihilation. However, in a companion paper [17] (hereafter Paper I), we calculated the DM-induced SZ effect for clusters. We showed that contrary to previous claims, the DM annihilation induced SZ effect in galaxy clusters is not as large as previously work [16], and there is little hope to detect it in the foreseeable future, which confirm the quantitative conclusion in [1]. Similar conclusion is also derived in a recent study [18]. In the present paper, we generalize our earlier work to the case of dSphs, for two DM candidates: the neutralino and the LDM.

The rest of the paper is organized as follows: in Section 2, we discuss our method of calculation. We first briefly review the equations for SZ calculation with a given electron distribution. Then we discuss the density distribution of the DM in dSphs. Lastly the production of electron $\mathrm{s}$ and positrons from DM annihilation and propagation of these $e^{ \pm}$are discussed. This differs somewhat from the case of clusters, as diffusion is more significant. In Section 3, we present the results of our numerical calculation. Finally we discuss our results and conclude in Section 4.

\section{Method of Calculation}

\subsection{The nonthermal SZ Effect}

For the calculation of the SZ effect, our method here is the same as in Paper I (see also [11). The spectral distortion of CMB after travelling through a population of electrons or positrons ${ }^{1}$ is given by

$$
\frac{\Delta T(x, \theta)}{T_{0}}=\frac{\left(e^{x}-1\right)^{2}}{x^{4} e^{x}} g(x) y(\theta),
$$

where $x=h \nu / k T_{0}$ is the dimensionless frequency of CMB photon, $T_{0}=2.725 \mathrm{~K}$ is the undistorted CMB temperature, $g(x)$ is the spectral distortion function, and $y(\theta)$ is the Comptonization parameter for angle separation $\theta$ from the center. The spectral distortion function is given by

$$
g(x)=\frac{m_{e} c^{2}}{\left\langle k T_{e}\right\rangle}\left[\int i_{0}\left(x e^{-s}\right) P_{1}(s) \mathrm{d} s-i_{0}(x)\right],
$$

where $i_{0}(x)=x^{3} /\left(e^{x}-1\right)$ is the Planckian distribution of CMB photons, $s=\ln \left(\nu^{\prime} / \nu\right)$ is the frequency shift of one photon after one scattering with $e^{ \pm}$. Here $P_{1}(s)$ is the frequency shift probability distribution after one scattering,

$$
P_{1}(s)=\int f_{e}(k) P_{s}(s, k) \mathrm{d} k,
$$

\footnotetext{
${ }^{1}$ As all the calculations are the same for electrons and positrons, in this paper, the subscript $e$ represents both $e^{+}$and $e^{-}$
} 
where $k$ is the dimensionless momentum (momentum in units of $\left.m_{e} c\right), P_{s}(s, k)$ is the probability of a photon to have a frequency shift $s$ when colliding with an electron with momentum $k, f_{e}(k)$ is the normalized momentum spectrum of electrons

$$
f_{e}(k)=\frac{1}{n_{e}} \frac{\mathrm{d} n_{e}}{\mathrm{~d} E_{e}} \frac{\mathrm{d} E_{e}}{\mathrm{~d} k}
$$

with $\frac{\mathrm{d} n_{e}}{\mathrm{~d} E_{e}}\left(E_{e}, r\right)$ the number density of $e^{ \pm}$per unit energy interval, which will be discussed in details in the next section when the dSphs and DM candidate are specified. The number density of non-thermal electrons is

$$
n_{e}(r)=\int_{E_{\min }}^{E_{\max }} \frac{\mathrm{d} n_{e}}{\mathrm{~d} E_{e}}\left(E_{e}, r\right) \mathrm{d} E_{e}
$$

with $E_{\max }$ and $E_{\min }$ the maximum energy and minimum energy of electrons produced by DM annihilation. The effective pressure of the non-thermal electrons can be defined as (see e.g. [19, 20])

$$
P_{e}=n_{e} \int_{k_{\min }}^{k_{\max }} f_{e}(k)\left(\frac{k^{2}}{3 \sqrt{1+k^{2}}}\right) m_{e} c^{2} \mathrm{~d} k
$$

with $k_{\max }=\sqrt{\left(E_{\max } / m_{e}\right)^{2}-1}$, and $k_{\min }=\sqrt{\left(E_{\min } / m_{e}\right)^{2}-1}$. In the relativistic limit, this definition approaches to the usual approximation

$$
P_{e} \approx \frac{1}{3}\langle E\rangle, \quad\langle E\rangle=\int_{k_{\min }}^{k_{\max }} f_{e}(k)\left(\sqrt{1+k^{2}}-1\right) m_{e} \mathrm{~d} p,
$$

in which $\langle E\rangle$ is the averaged kinetic energy. When DM annihilation is the only source of $e^{ \pm}$, the upper limit of the integrals are set to be $E_{\max }=m_{\mathrm{DM}}$ and $k_{\max }=\sqrt{\left(m_{\mathrm{DM}} / m_{e}\right)^{2}-1}$. In some analyses [16], $E_{\text {min }}$ is set to be $0.01 m_{\mathrm{DM}}$, which was considered to be a good approximation as it is small enough comparing with the peak of the energy spectrum of continuous electrons $\left(E_{\text {peak }}=1 / 20 m_{\mathrm{DM}}\right)$ as shown in [0]. However, for the non-thermal SZ effect in dwarf galaxies, the contribution of electrons with $E<0.01 m_{\mathrm{DM}}$ to the final signal is still significant. Therefore, we use Eq. (2.4) which gives a more accurate expression for the contribution of trans-relativistic electrons and set $E_{\min }=m_{e}$ as no artificial cut off is necessary. Note however that even though this expression itself is accurate, there could still be significant error in the expression of $f_{e}(k)$ used for calculation at the lowest energies, see the discussion in $\S 2.4$ on $e^{+} e^{-}$production from DM annihilations.

The contribution to Comptonization parameter by DM annihilation is given by the line-of-sight integral of effective gas pressure through the $e^{ \pm}$cloud:

$$
y=\frac{\sigma_{T}}{m_{e} c^{2}} \int P_{e} \mathrm{~d} l,
$$

where $\sigma_{T}$ is the Thomson cross section.

The observed effective temperature change due to $\mathrm{SZ}_{\mathrm{DM}}$ is an average of the temperature change within a beam

$$
\Delta T_{o b s}(\mathbf{n}, \theta)=\int B_{\mathbf{n}}\left(\mathbf{n}^{\prime}, \theta\right) \Delta T\left(\mathbf{n}^{\prime}\right) \mathrm{d} \mathbf{n}^{\prime},
$$


where $B_{\mathbf{n}}\left(\mathbf{n}^{\prime}, \theta\right)$ is the beam profile in the direction $\mathbf{n}^{\prime}$ for a beam centered in the direction $\mathbf{n}$ with beam size $\theta$. For illustration, we have made our calculation with a tophat beam profile and different beam widths.

\section{2 dark matter distribution}

In dSphs, DM dominates these systems both in the inner parts and outskirts. Similar as in Paper I, we consider the following three types of DM density profiles:

$$
\begin{aligned}
& \rho(r)=\frac{\rho_{\mathrm{s}}}{\left(1+r / r_{s}\right)\left[1+\left(r / r_{s}\right)^{2}\right]} \text { (hereafter B95, Ref. 21]), } \\
& \rho(r)=\frac{\rho_{\mathrm{s}}}{\left(r / r_{s}\right)\left(1+r / r_{s}\right)^{2}} \quad \text { (hereafter NFW, Ref. 22]), } \\
& \rho(r)=\frac{\rho_{\mathrm{s}}}{\left(r / r_{s}\right)^{1.5}\left[1+\left(r / r_{s}\right)^{1.5}\right]} \text { (hereafter M99, Ref. 23]). }
\end{aligned}
$$

They show similar behaviors $\left(\sim r^{-3}\right)$ at large radii, but differs significantly near the center of the halo. We employ the B95, NFW and M99 profiles to represent the non-cuspy, moderately cuspy and strongly cuspy profiles of DM halos respectively.

The density profile is truncated at a small radius where we assume the annihilating rate matches the in-falling rate of DM [24]. Within this radius, the density is kept at a constant value as

$$
\rho_{\max }=3 \times 10^{18} \frac{m_{\mathrm{DM}}}{100 \mathrm{GeV}} \frac{10^{-26} \mathrm{~cm}^{2} \mathrm{~s}^{-1}}{\langle\sigma v\rangle} \mathrm{M}_{\odot} \mathrm{kpc}^{-3} .
$$

The density distribution of DM could in principle be determined from the velocity dispersion of stars. However, the uncertainty is large, and in the literature the required data is not always complete or easy to use. Parameters such as the characteristic density $\rho_{s}$ and radius $r_{s}$ are chosen to be set by adopting specific density profile. Another generally used parameters are the virial mass $\mathrm{M}_{\mathrm{vir}}$ and concentration parameters $c_{\mathrm{vir}}$. The virial radius of a DM halo is defined as

$$
r_{\mathrm{vir}}=\left(\frac{\mathrm{M}_{\mathrm{vir}}}{(4 \pi / 3) \Delta \rho_{c}}\right)^{1 / 3}
$$

where $\Delta$ is the overdensity, and $\rho_{\mathrm{c}} \approx 139 \mathrm{M}_{\odot} \mathrm{kpc}^{-3}$ is the critical density of the universe. For the $\Lambda$ CDM universe, $\Delta \approx 18 \pi^{2}+82 x-39 x^{2}$ with

$$
x=\Omega_{M}(z)-1=-\frac{\Omega_{\Lambda}}{\Omega_{M}(1+z)^{3}+\Omega_{\Lambda}}
$$

is found to be a good approximation [25]. The concentration parameter $c_{\mathrm{vir}}$ is defined as

$$
c_{\mathrm{vir}}=\frac{r_{\mathrm{vir}}}{r_{-2}}
$$

where $r_{-2}$ refers to the radius at which $\left.\frac{\mathrm{d}\left(r^{2} \rho\right)}{d r}\right|_{r=r_{-2}}=0$. The concentration parameter $c_{\mathrm{vir}}$ relates $r_{\text {vir }}$ and the density profile parameter as [26]

$$
r_{\mathrm{s}}^{\mathrm{B} 95}=\frac{r_{\mathrm{vir}}}{1.52 c_{\mathrm{vir}}}, r_{\mathrm{s}}^{\mathrm{NFW}}=\frac{r_{\mathrm{vir}}}{c_{\mathrm{vir}}}, r_{\mathrm{s}}^{\mathrm{M} 99}=\frac{r_{\mathrm{vir}}}{0.63 c_{\mathrm{vir}}} .
$$


Simulations show that $c_{\text {vir }}$ and $M_{\text {vir }}$ are often correlated. Here we use a power-law relation between $c_{\mathrm{vir}}$ and $M_{\mathrm{vir}}$ as suggested in Ref. [27]

$$
c\left(M_{\mathrm{vir}}\right)=c_{0} \times\left(\frac{M_{\mathrm{vir}}}{10^{14} h^{-1} \mathrm{M}_{\odot}}\right)^{\alpha},
$$

where $c_{0}=9.6$ and $\alpha=-0.1$ for a $\Lambda$ CDM cosmology. With this assumption, the DM distribution is fixed once $M_{\text {vir }}$ is given.

The halo of the dSphs would, however, be disrupted by the tidal force of the host galaxy (Milky Way), as a result part of the DM at the outer edge of the halo would be removed. To account for this effect, we calculate the tidal radius, which is given by the Roche criterion 28

$$
\frac{M_{\mathrm{dSph}}\left(r_{t}\right)}{r_{t}^{3}}=\frac{M_{\mathrm{MW}}\left(\mathrm{D}-\mathrm{r}_{\mathrm{t}}\right)}{\left(\mathrm{D}-\mathrm{r}_{\mathrm{t}}\right)^{3}}
$$

where $\mathrm{D}$ is the distance between the Galactic center and the dSph, $r_{t}$ is the tidal radius of the dSph and $M(r)$ is the mass inside radius $r$. The results depend on the choice of the profile for Milky Way halo. Here we adopted the isothermal power law model [29] for the Milky Way

$$
\rho_{\text {iso }}=\frac{v_{a}^{2}}{4 \pi G} \frac{3 r_{s}^{2}+r^{2}}{\left(r_{s}^{2}+r^{2}\right)^{2}}
$$

with $r_{s}=10 \mathrm{kpc}$ and $v_{a}=220 \mathrm{~km} \mathrm{~s}^{-1}$ as in [16, 30].

In Table 1, we list the parameters for a sample of 7 nearby dSphs, which were obtained by fitting the line-of-sight velocity dispersion profile of the stars [31]. NFW profile was assumed when deriving these parameters. Strictly speaking, these parameters should not be used for other halo profiles. To be self-consistent, one should re-estimate the virial mass with different halo profiles. However, since there are many uncertainties in both the observation and theoretical modeling, and the results of this paper should be regarded as an order-of-magnitude estimate, we will just use the same virial mass for M99 and B95 profiles but re-evaluate the halo parameters.

The nearby and relatively large Draco dwarf represents one of the best candidates for searching for DM in dSphs, and it has been studied extensively with many observations at different wavelengths. Since the basic properties of the SZ effects in different dSphs are qualitatively similar to each other, we will take the Draco dwarf as the prime example in the following discussions.

\section{$2.3 e^{ \pm}$production from DM annihilation}

As the SZ effect produced by positrons and electrons can not be distinguished, here we use $e$ to refer both $e^{+}$and $e^{-}$. The $e^{ \pm}$source function from DM annihilation can be written as

$$
Q_{e}(E, r)=\frac{\langle\sigma v\rangle}{2 m_{\mathrm{DM}}^{2}} \sum_{f} \frac{\mathrm{d} N_{e}^{f}}{\mathrm{~d} E_{e}}(E) B_{f} \rho^{2}(r),
$$

where $\langle\sigma v\rangle$ is the velocity-weighted annihilation cross section, $m_{\mathrm{DM}}$ is the DM particle mass, $\rho(r)$ is energy density of DM, and $\mathrm{d} N_{e} / \mathrm{d} E_{e}$ is the number of electrons produced per 
Table 1: The dSphs parameters used in this paper. NFW profile of dSph is assumed when calculating $r_{s}, \rho_{s}$ and $r_{t}$. The distance and virial mass data are mostly taken from Ref. [31], other parameters are calculated as outlined below. For Ursa Minor, which was not included in Ref. 31, we take the mass as to be the same as that of Draco.

\begin{tabular}{|c|c|c|c|c|c|}
\hline Name & $\frac{\mathrm{D}}{|\mathrm{kpc}|}$ & $\frac{M_{\mathrm{vir}}}{10^{8} \mathrm{M}_{\odot}}$ & $\begin{array}{c}\frac{\rho_{s}}{10^{8} \mathrm{M}_{\odot} / \mathrm{kpc}^{3}} \\
n^{2}\end{array}$ & $\frac{r_{s}}{|\mathrm{kpc}|}$ & $\frac{r_{t}}{|\mathrm{kpc}|}$ \\
\hline Draco & 80 & 40 & 0.82 & 1.2 & 9.9 \\
\hline LeoI & 250 & 10 & 1.2 & 0.64 & 16.7 \\
\hline Fornax & 138 & 10 & 1.2 & 0.64 & 10.3 \\
\hline LeoII & 205 & 4 & 1.5 & 0.43 & 10.6 \\
\hline Carina & 101 & 2 & 1.8 & 0.32 & 4.8 \\
\hline Sculptor & 79 & 10 & 1.2 & 0.64 & 6.5 \\
\hline Sextans & 86 & 3 & 1.6 & 0.38 & 4.8 \\
\hline Ursa Minor & 66 & 40 & 0.82 & 1.2 & 8.6 \\
\hline
\end{tabular}

annihilation per energy interval. In the following, we discuss two types of DM particles: neutralino in the supersymmetric model, and the LDM.

Neutralino: We consider a neutralino with the typical values of parameters $\langle\sigma v\rangle=$ $3 \times 10^{26} \mathrm{~cm}^{3} \mathrm{~s}^{-1}$ and $m_{\mathrm{DM}}=100 \mathrm{GeV}$. The direct channel to $e^{+} e^{-}$is generally suppressed for neutralino, so electrons are in most cases produced from the cascades of the annihilation final-state particles such as heavy leptons, quarks and gauge bosons [2]. The spectra of electrons can be different from each other for different annihilation modes. We use the package DarkSUSY [32] to calculate the final-state spectra of electrons. Our fiducial annihilation mode is assumed to $W^{+} W^{-}$.

In Fig. 1, we plot the resulting electron energy spectrum for several annihilation modes. In the same figure, we also plot the parametrized fit of spectra used in [16] for comparison. As we can see from the figure, the parametrization fits the DarkSUSY spectrum well above $1 \mathrm{GeV}$. However below $0.1 \mathrm{GeV}$ this analytical form deviates significantly from the DarkSUSY results.

LDM: Inspired by the excess of $511 \mathrm{keV}$ lines at the Galactic center observed by SPI/INTEGRAL 33, 34, it was proposed that a light DM particle with mass $1-100 \mathrm{MeV}$ may be responsible for the data [35]. To allow the production of positrons through its annihilation, one needs $m_{\mathrm{DM}}>m_{e}$. The mass of LDM should also be less than $\sim 100 \mathrm{MeV}$ to avoid producing too many $\gamma$-ray photons from $\pi$ final state. The model has since been further constrained by a number of more detailed considerations on its phenomenology [36, 37, 38, 39, 40]. For example, a small fraction of the energetic positrons produced in the annihilation could be directly annihilated into energetic $\gamma$-rays (in-flight annihilation), and the limit on $\gamma$-rays yields stringent limits of $m_{\mathrm{DM}}<3 \mathrm{MeV}$ [38] or $m_{\mathrm{DM}}<7.5 \mathrm{MeV}$ [39], depending on the ionization state of the interstellar medium in the Galactic center. A comparable bound of $m_{\mathrm{DM}}<7 \mathrm{MeV}$ was derived using $\mathrm{CMB}$ data by considering the effect of LDM annihilation during the recombination history [40].

Here, as in Paper I, we assume the mass of the LDM is $5 \mathrm{MeV}$, which is consistent 


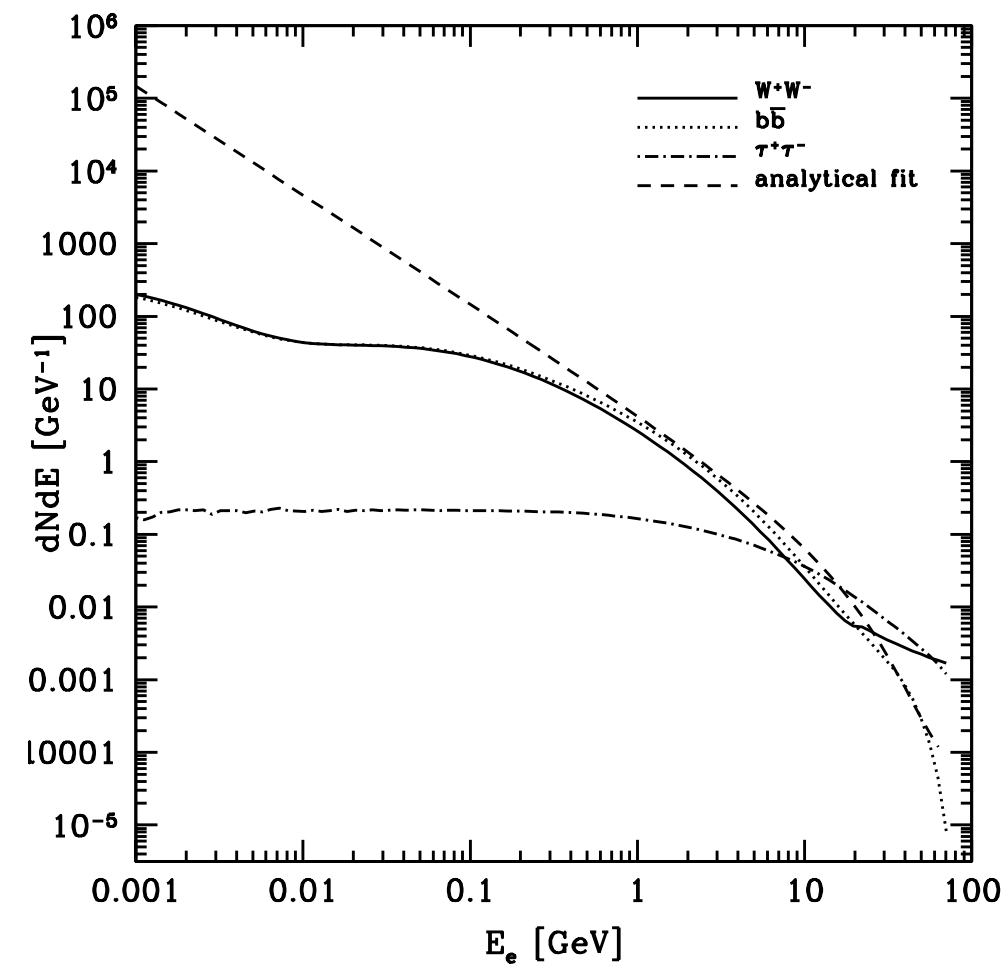

Figure 1: Electron yield spectra $\mathrm{d} N / \mathrm{d} E$ for $W^{+} W^{-}$(solid curve), $b \bar{b}$ (dotted curve) and $\tau^{+} \tau^{-}$(dotdashed curve) annihilation modes for neutralino with mass $m_{\chi}=100 \mathrm{GeV}$. The dashed curve refers to the analytical fit adopted in [16].

with the limits obtained in [39, 40]. It is slightly beyond the constraint of [38], but note that the SZ effect here would be stronger for smaller DM mass, so our choice is actually conservative. The cross section derived from the flux of the $511 \mathrm{keV}$ line emission from the bright Galactic bulge region is $\langle\sigma v\rangle_{\mathrm{LDM}} \sim 10^{30}\left(m_{\mathrm{DM}} / \mathrm{MeV}\right)^{2} \mathrm{~cm}^{3} \mathrm{~s}^{-1}$. Thus for our mass choice of LDM we have $\langle\sigma v\rangle=2.5 \times 10^{-29} \mathrm{~cm}^{3} \mathrm{~s}^{-1}$. The recent observations of the spatial morphology of the line emission by INTEGRAL [41] found a significant asymmetry in the disk emission with a resemblance to the observed distribution of low-mass X-ray binaries in the hard state, which indicates that these X-ray binaries might be the main sources. However, it is worthwhile to note that the large bulge-to-disk ratio $(3 \sim 9)$ is not easily achieved even with the assumption that a large fraction of the disk positrons is transported via the regular magnetic field of the Galaxy into the bulge and annihilate there, so the LDM model can not be excluded yet. Our assumption about the annihilating cross section may be regarded as the upper limit of LDM contribution to the observed 511 $\mathrm{keV}$ line. We assume the produced $e^{ \pm}$have a monochrome spectra $\mathrm{d} N_{e} / \mathrm{d} E_{e}=\delta\left(E-m_{\mathrm{DM}}\right)$ in this case.

\section{$2.4 e^{ \pm}$propagation in dwarf galaxies}

DM annihilation injects electrons and positrons in the galaxy halo at a constant rate. The propagation of these charged particles in the tangled magnetic field can be modeled by 
diffusion. They also lose energy by radiation during this process. As a result, the $e^{ \pm}$ spectrum satisfies the following transport equation 42]:

$$
\frac{\partial}{\partial t} \frac{\mathrm{d} n_{e}}{\mathrm{~d} E_{e}}=\nabla\left[D(r, E) \nabla \frac{\mathrm{d} n_{e}}{\mathrm{~d} E_{e}}\right]+\frac{\partial}{\partial E}\left[b(r, E) \frac{\mathrm{d} n_{e}}{\mathrm{~d} E_{e}}\right]+Q_{e}(r, E),
$$

where $\mathrm{d} n_{e} / \mathrm{d} E_{e}$ is the number density of $e^{ \pm}$per unit energy interval, $D(E, r)$ is the diffusion coefficient, $b(E, r)=-\mathrm{d} E_{e} / \mathrm{d} t$ represents the energy loss rate, and $Q_{e}(E, r)$ is the source function. For simplicity, we assume that $D$ and $b$ are independent of spatial location. The diffusion coefficient $D(E)$ is assumed to have a power law dependence on energy $E$ and magnetic field $B: D(E)=D_{0}(E / B)^{\delta}$, with $\delta=1 / 3$ [16], although it is not clear to what extent this relation could be extrapolated. Due to the much smaller scale of uniformity of the magnetic field in dwarf galaxies, we set $D_{0}=3.1 \times 10^{26} \mathrm{~cm}^{2} \mathrm{~s}^{-1}$, which is lower than the value in the Milky Way [1, 43]. We also set $B_{\mu}=1 \mu \mathrm{Gs}$ as our fiducial value of magnetic field.

The energy loss rate is given by $b(E)=b_{\mathrm{ICS}}(E)+b_{\mathrm{syn}}(E)+b_{\text {ion }}(E)$, i.e.

$$
\frac{b(E)}{10^{-17} \mathrm{GeV} \mathrm{s}^{-1}}=2.5 \times\left(\frac{\beta E}{1 \mathrm{GeV}}\right)^{2}+0.25 \times\left(\frac{B_{\mu}}{1 \mu G} \frac{\beta E}{1 \mathrm{GeV}}\right)^{2}+2 \times \frac{N_{\mathrm{H}}}{1 \mathrm{~cm}^{-3}}[\ln (\Gamma)+6.6],
$$

where $N_{\mathrm{H}} \simeq 1.3 \times 10^{-6} \mathrm{~cm}^{-3}$ is the number density of neutral gas in dSphs, $\Gamma$ and $\beta$ are the Lorentz factor and the velocity of $e^{ \pm}$respectively.

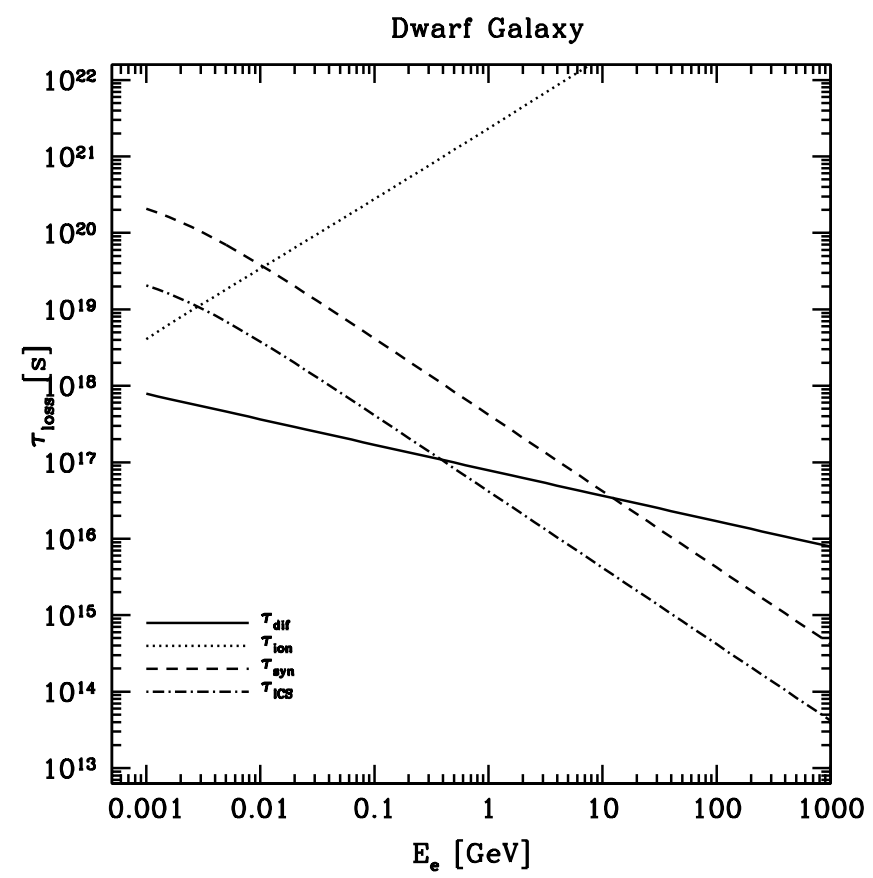

Figure 2: Comparison of the time scales for various energy loss mechanisms and diffusion in a dSph galaxy with size $r_{h}=1.6 \mathrm{kpc} . B=1 \mu \mathrm{Gs}$ and $N_{\mathrm{H}}=1.3 \times 10^{-6} \mathrm{~cm}^{-3}$ are assumed.

The characteristic time scales for different energy loss mechanisms and spatial diffusion are plotted in Fig. 2. The energy loss time-scale is defined as $\tau_{\text {loss }}=E / b(E)$, and the 
diffusion time-scale is $\tau_{\text {dif }} \approx r_{h}^{2} / D(E)$. We clearly see that the ionization loss has negligible effects, except below $\mathrm{MeV}$ energies. However, as the size of the dwarf galaxy is small, spatial diffusion could dominate at this energy range. Synchrotron emission and inverse Compton scattering (ICS) off the CMB photons are important for high energy electrons.

The transport equation (2.19) could be solved with the Green's function method as described in [1]. For time-independent source function, the equilibrium solution has the form

$$
\frac{\mathrm{d} n_{e}}{\mathrm{~d} E}(r, E)=\frac{1}{b(E)} \int_{E}^{m_{\mathrm{DM}}} \mathrm{d} E^{\prime} \widehat{G}(r, \Delta v) Q_{e}\left(r, E^{\prime}\right) .
$$

where $\Delta v\left(E, E^{\prime}\right)=\int_{E}^{E^{\prime}} \mathrm{d} \epsilon D(\epsilon) / b(\epsilon)$ is the mean scale of diffusion covered by an electron while losing energy from $E^{\prime}$ to $E$. For a spherical symmetric system with free escape boundary condition at $r_{h}$ (i.e. assuming the magnetic field is so weak that beyond this region the electrons are not confined), the Green's function is given by

$\widehat{G}(r, \Delta v)=\frac{1}{\sqrt{4 \pi \Delta v}} \sum_{n=-\infty}^{+\infty}(-1)^{n} \int_{0}^{r_{h}} \mathrm{~d} r^{\prime} \frac{r^{\prime 2} \rho^{2}\left(r^{\prime}\right)}{r r_{n}^{\prime} \rho^{2}(r)}\left[\exp \left(-\frac{\left(r-r_{n}^{\prime}\right)^{2}}{4 \Delta v}\right)-\exp \left(-\frac{\left(r+r_{n}^{\prime}\right)^{2}}{4 \Delta v}\right)\right]$,

where $r_{n}^{\prime}=(-1)^{n} r^{\prime}+2 n r_{h}$ is the location of $n$th "charge" image for $r_{h}$ (c.f. Ref. [13]). The value of $r_{h}$ is generally adopted as twice of the radius of the stellar component, typically a few kpc for local dSphs.

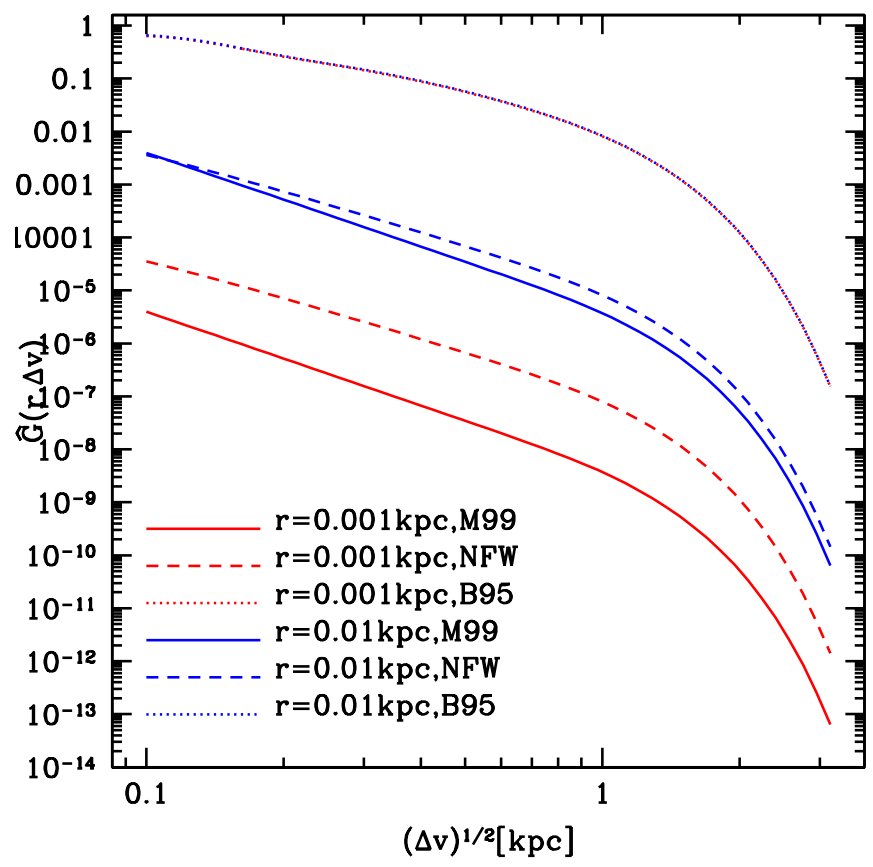

Figure 3: The Green's function $\widehat{G}$ varies with respect to $\sqrt{\Delta v}$ for two different radii $r=0.001 \mathrm{kpc}$ and $r=0.1 \mathrm{kpc}$ and for three density profiles: B95, NFW and M99.

The behavior of the Green's function $\widehat{G}(r, \Delta v)$ is shown in Fig. 3. We plot $\widehat{G}$ as a function of $\sqrt{\Delta v}$ for two different radii and for the three density profiles described in Sec. 
2.2. As expected, the Green's function decreases as the diffusion length $\sqrt{\Delta v}$ increases. Near the center of the galaxy, however, we find $\widehat{G} \ll 1$ as represented by the $r=0.001 \mathrm{kpc}$ curves for the M99 and NFW profiles, indicating that the diffusion effect is significant and it will affect the propagated spectrum. We can also see that for more cuspy density profile, the decrement in $\widehat{G}$ is also larger.

When spatial diffusion can be neglected, $\widehat{G} \sim 1$, we obtain the solution for no diffusion or in situ energy loss:

$$
\left.\frac{\mathrm{d} n_{e}}{\mathrm{~d} E}(r, E)\right|_{\mathrm{nodif}}=\frac{1}{b(E)} \int_{E}^{m_{\mathrm{DM}}} \mathrm{d} E^{\prime} Q_{e}\left(r, E^{\prime}\right)
$$

The spatial distribution of $e^{ \pm}$then traces the source function, which is proportional to the density square of DM. However, for dSphs this approximation is generally invalid.

\section{Results}

\subsection{Distribution of electrons}

To take the diffusion effect into account, Ref. [13] also proposed an analytic approximate solution of the transport equation

$$
\frac{\mathrm{d} n_{e}}{\mathrm{~d} E}(r, E) \approx\left[Q_{e}(E, r) \tau_{\text {loss }}\right] \times \frac{V_{s}}{V_{s}+V_{o}} \times \frac{\tau_{D}}{\tau_{D}+\tau_{\text {loss }}},
$$

where $V_{s} \propto r_{h}^{3}$ is the volume occupied by the DM source ${ }^{2}$, and $V_{o} \propto \lambda^{3}(E)$ is the volume occupied by a diffusing electron which travels a distance $\lambda(E) \approx\left[D(E) \times \tau_{\text {loss }}(E)\right]^{1 / 2}$ before losing a significant fraction of its initial energy. This changes the total number of electrons, but does not change the shape of its density profile. Here we call this model as the "approximate solution".

We plot in Fig. 4 the total number density distribution of $e^{ \pm}$produced by neutralino annihilation for the case of dSph Draco with an assumed M99 profile. Our full solution obtained with the Green's function method is shown as the solid curve, while the "approximate solution" is shown as the dotted one. The dot-dashed curve below the solid one is the result obtained with $E_{\min }=0.01 m_{\mathrm{DM}}$. We see that the non-thermal electrons with $E<0.01 m_{\text {DM }}$ still make up a significant fraction of the total density. Previous analysis on $\mathrm{SZ}_{\mathrm{DM}}$ in dwarf galaxies in Ref. [16] predicted $\mu \mathrm{K}$-level temperature distortion within 1 arcsec for strongly cusped (M99) dark halo. They employed the approximation

$$
n_{e}(r)=\left.\int_{0.01 m_{\mathrm{DM}}}^{m_{\mathrm{DM}}} \frac{\mathrm{d} n_{e}}{\mathrm{~d} E}(r, E)\right|_{\text {nodif }} \mathrm{d} E .
$$

This "no-diffusion solution" is shown as the dashed line. It is apparent that there are significant differences between the full solution and the simplified solution or the approximate solution. The $e^{ \pm}$density profile of our full solution has a significantly shallower slope than

\footnotetext{
${ }^{2}$ As the galactic magnetic field has a limited extent, here we define $r_{h}$ as the radius of diffusion zone instead of the tidal radius of the dark halo.
} 


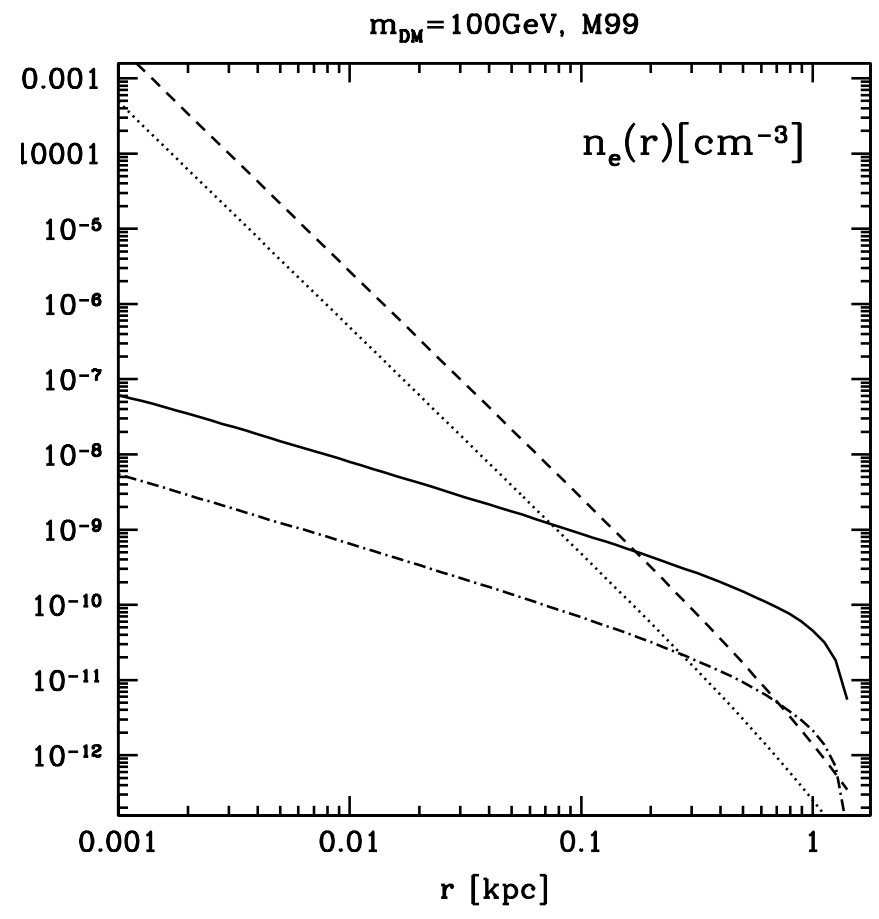

Figure 4: The $e^{ \pm}$density $n_{e}(r)$ from neutralino annihilations in the Draco dSph. The solid and dot-dashed curves are our full solutions with $E_{\min }=m_{e}$ and $0.01 m_{\mathrm{DM}}$ respectively. The dotted curve is the result of the "approximate solution", and the dashed curve is the result of the "nodiffusion solution" as described in Eq. (3.2).

the "approximate solution". Thus in the inner region, the full solution predicts much lower electron number density than either the "no-diffusion solution" or the "approximate solution". We can understand this by noting that diffusion could remove electrons in the inner region and spread them in a larger region. Although the "no-diffusion solution" attempts to account for the effect of diffusion by reducing the total number density of electrons, it reduces the electron density by the same factor at all radii. Actually the inner region will be affected much more significant than the outer regions. Also, for more cuspy halos, the scale over which $\rho^{2}(r)$ has a significant gradient is smaller, resulting in more remarkable loss of electrons due to diffusion.

In Fig. 5 we plot the density distribution of electrons produced by DM annihilation for different DM halo density profiles, all of which are obtained with the Green's function method. As expected, the strongly cuspy M99 profile produces the largest $e^{ \pm}$density, followed by NFW profile and then B95 profile. In the center, the density of electrons of M99 profile is several orders of magnitude higher than that of NFW profile. Thus, the density profile of DM halo is crucial in determining the strength of the $\mathrm{SZ}_{\mathrm{DM}}$ signal.

\subsection{The $\mathrm{SZ}_{\mathrm{DM}}$ signal}

The expected spectral distortion of CMB due to the energetic $e^{ \pm}$from DM annihilation observed with $1^{\prime \prime}$ beam aimed at the center of the Draco dSph are shown in Fig. 6. The left panel is for a $100 \mathrm{GeV}$ neutralino, and right panel is for a $5 \mathrm{MeV}$ LDM. The three curves 


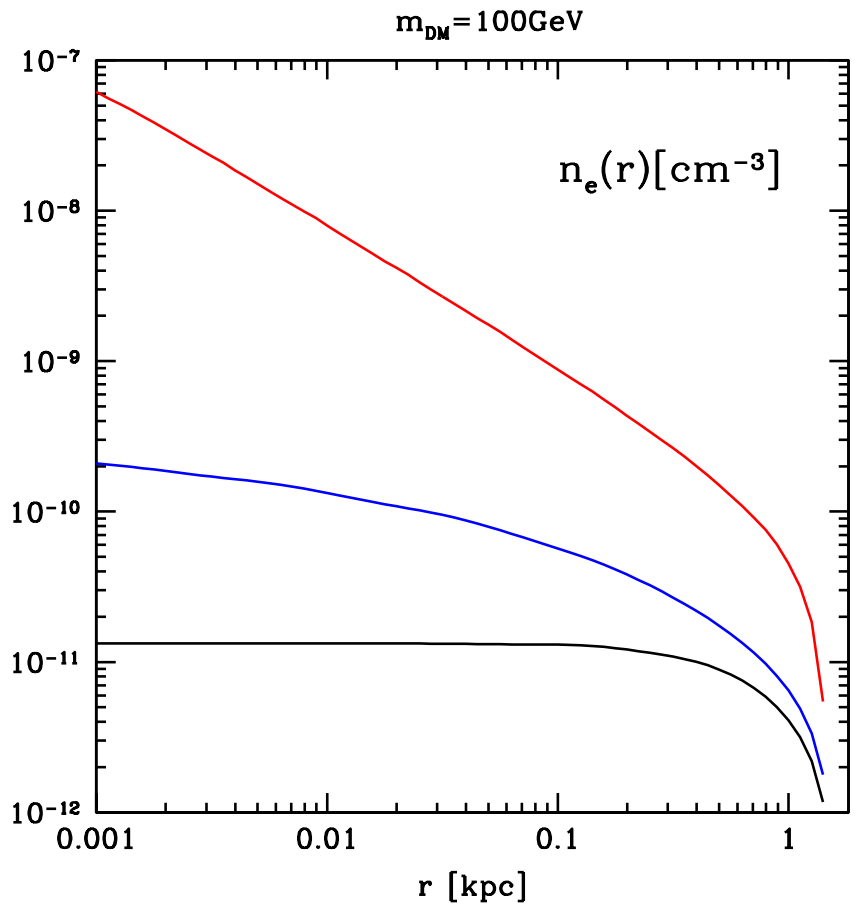

Figure 5: The $e^{ \pm}$density for different halo profiles assumed The red curve is the result for M99 profile, blue for NFW and black for B95.
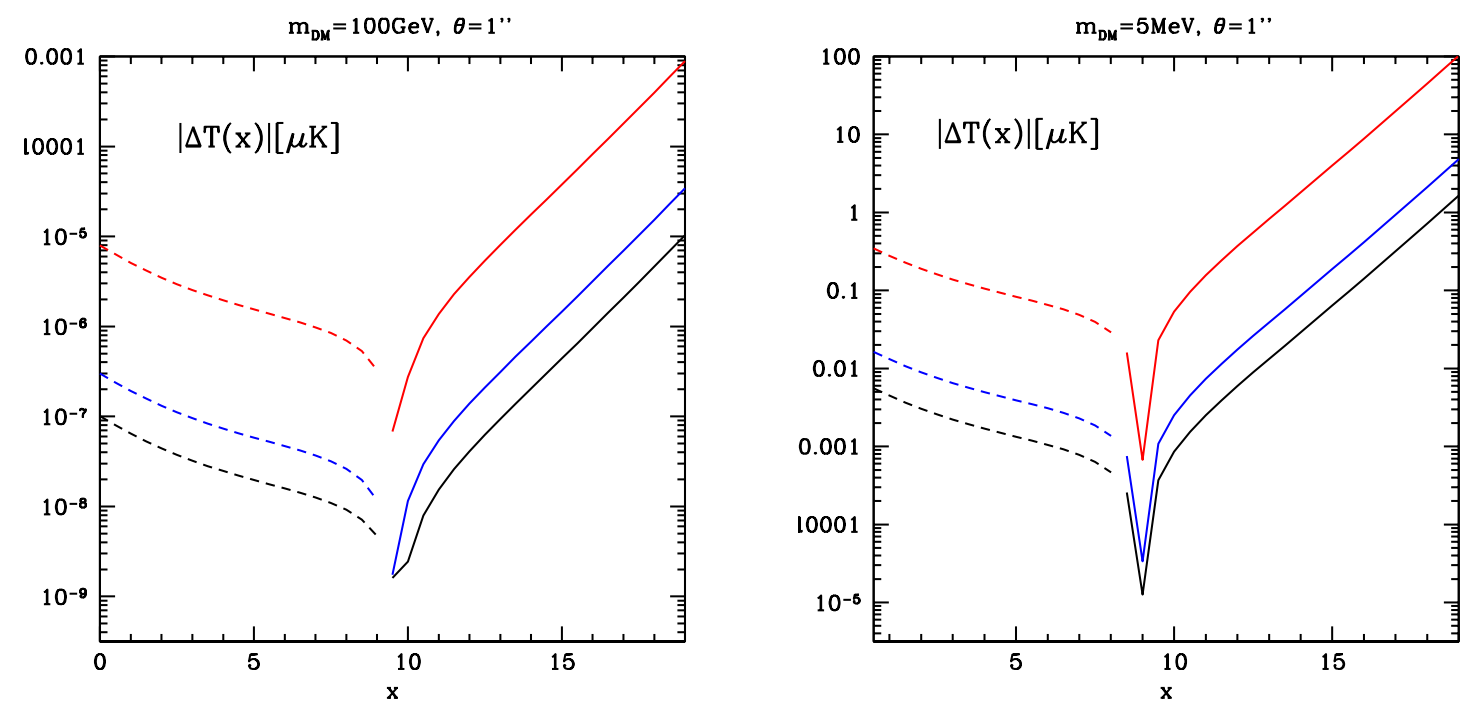

Figure 6: The SZ effect induced by the neutralino (left) and LDM (right) annihilations. The result for M99 profile (red), NFW profile (blue) and B95 profile (black) are shown.

in the figure are for M99, NFW and B95 DM halo profiles respectively. As expected, the strongly cuspy profile (M99) yields greater temperature distortion, while NFW and B95 profiles yield curves which are similar but with less magnitude. We plotted only the absolute value of the temperature distortion in this figure, note that at low frequency (small $x$ ) we have $\Delta T<0$, while at high frequency (large $x$ ) $\Delta T>0$. As a result the magnitude 

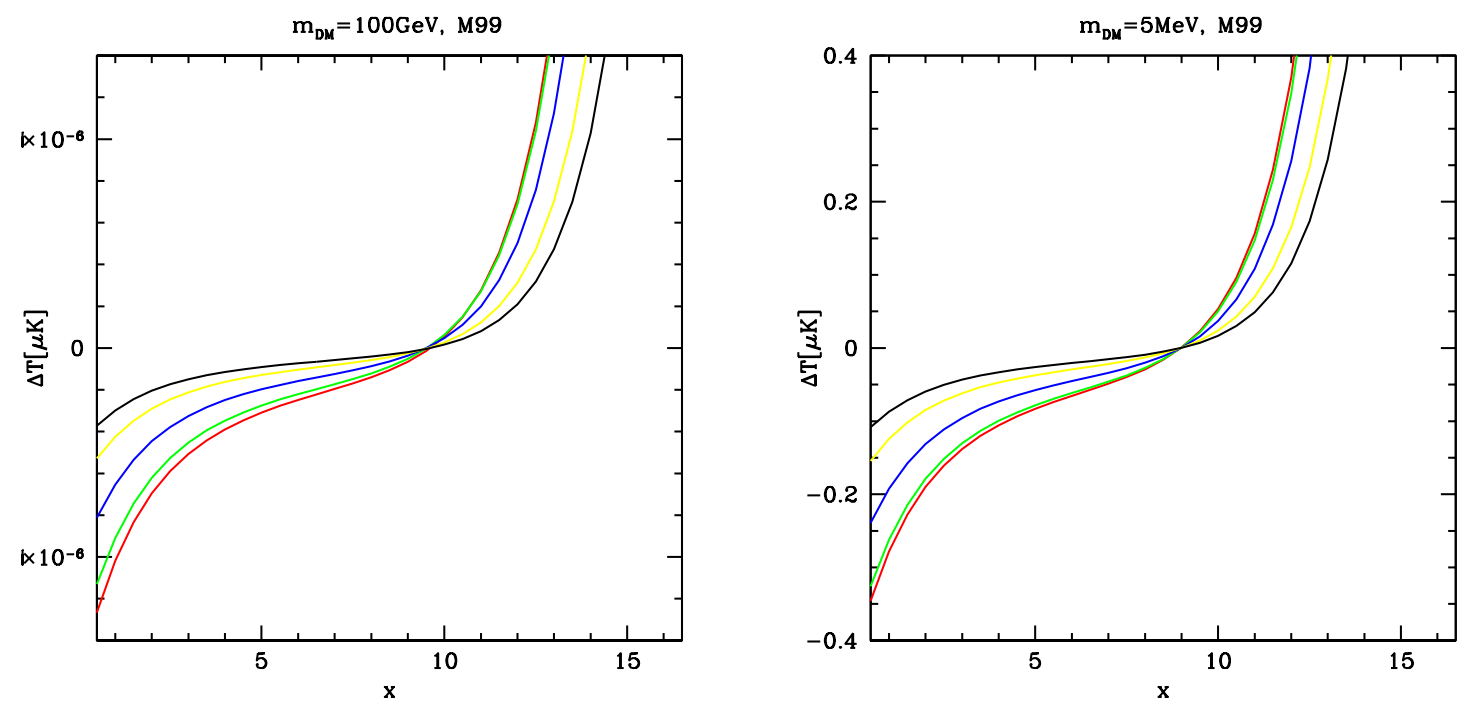

Figure 7: Spectral Distortion as a function of $x=h \nu / k T_{\mathrm{CMB}}$ for the case of Draco, with beam size ranging from $1^{\prime \prime}$ (red), $6^{\prime \prime}$ (green), $1^{\prime}$ (blue), $6^{\prime}$ (yellow) to $15^{\prime}$ (black).

of the temperature distortion appears to drop in the middle of the figure, where it reverses its sign. Unlike the case of the thermal SZ effect, this null point is not always fixed at 217 $\mathrm{GHz}$, but varying slightly.

The effects of different beam sizes are illustrated in Fig. 7. The red, green, blue, yellow and black curves are the observed temperature distortion for the beam size $1^{\prime \prime}, 6^{\prime \prime}, 1^{\prime}, 6^{\prime}$ and $15^{\prime}$ respectively. The smaller beam probes a smaller region around the center, hence the $\mathrm{SZ}_{\mathrm{DM}}$ signal is larger.

We find that the magnitude of the signal is much smaller than the previous estimate in the literature (e.g. Ref.[16]). In Fig. 8 we plot the $\mathrm{SZ}_{\mathrm{DM}}$ signal as a function of the beam width at $x=0.616(\nu=35 \mathrm{GHz})$ which is similar to the corresponding value in Ref. [16]. We also take the same values as $m_{\mathrm{DM}}=10 \mathrm{GeV},\langle\sigma v\rangle=1.0 \cdot 10^{-26} \mathrm{~cm}^{3} \mathrm{~s}^{-1}$ in order to have a direct comparison. The solid line is for DarkSUSY calculated electron injection spectrum with Green's function solution of the propagation equation. Other lines use the analytical form of the electron spectra which were shown as the dashed line in Fig. 1. The dot-dashed line in Fig. 8 is the result from our full solution with the Green's function, while the dotted line is for the "approximate solution", and the dashed line is for the "nodiffusion solution" respectively. Due to the large difference in the low energy electrons, the resulting $\mathrm{SZ}_{\mathrm{DM}}$ signals are quite different between the DarkSUSY and the analytic spectra. On the other hand, if one neglects diffusion or only treats it as a global reduction in the total number of electrons within the halo as were done in previous works, the signal could be much higher near the center of the halos. With the diffusion effect included, however, the brightness temperature only increases slightly near the center. Only when the beam width become large enough, which is comparable with the region occupied by the stellar component, the approximate model can be used. For the $10 \mathrm{GeV}$ neutralino, even for the M99 profile the expected signal in our fiducial model (shown as the solid line in Fig. 8) 


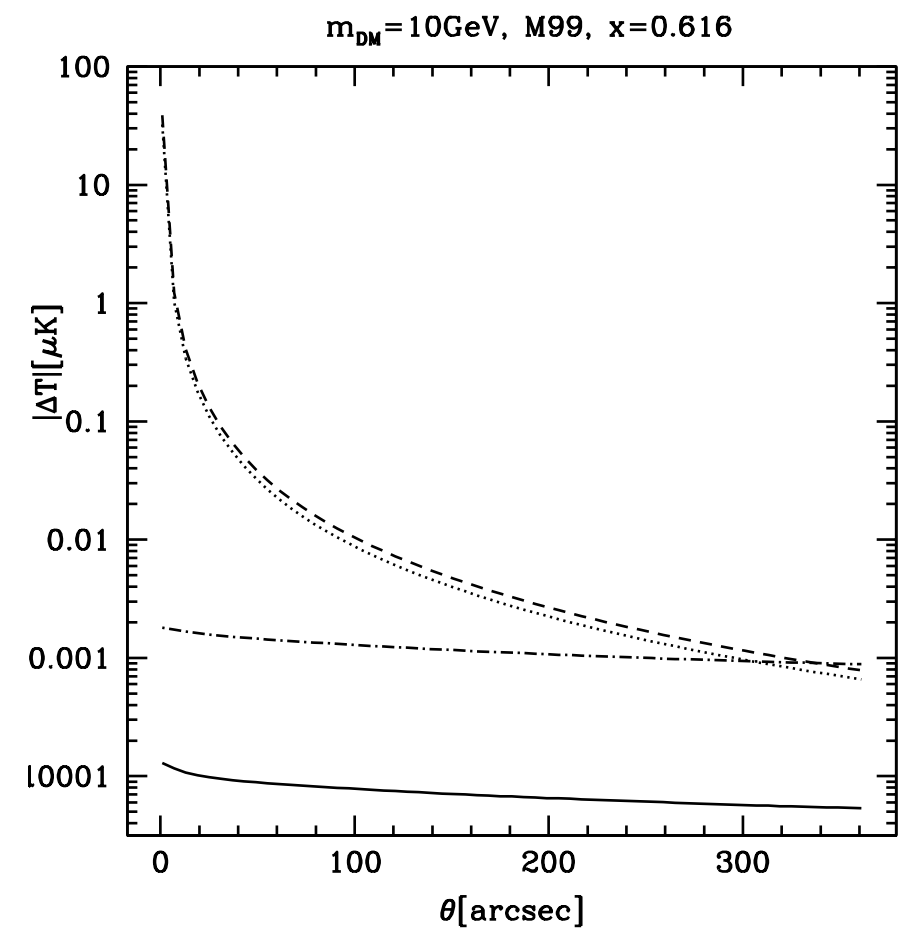

Figure 8: $\mathrm{SZ}_{\mathrm{DM}}$ signal as a function of observing beam width $\theta$. The solid line is our fiducial model with DarkSUSY electron spectra. Other lines use analytical spectra, with different approach to solve the transport equation: Green's function solution (dot-dashed), "approximate solution" (dotted), and "no-diffusion solution" (dashed) respectively.

at low frequency is well below $10^{-3} \mu \mathrm{K}$, which is not observable with the current or next generation instruments. At the high frequency end, the signal is higher, but it is difficult to observe from most sites on the ground. Furthermore, although the dSphs do not have a thermal SZ background, such small signal might be swamped by other foreground or background noises.

Finally, we illustrate in Fig. 9 the effect of different diffusion parameters in $D(E)$ which are not yet well-constrained. We plot the spectral distortion for three groups of $D_{0}$ and $\delta$. The solid line corresponds to our benchmark value $D_{0}=3.1 \times 10^{26} \mathrm{~cm}^{2} \mathrm{~s}^{-1}$ and $\delta=1 / 3$. The dot-dashed line is for the same $\delta$ but $D_{0}=1.0 \times 10^{27} \mathrm{~cm}^{2} \mathrm{~s}^{-1}$. The dashed line adopts the same $D_{0}$ but $\delta=2 / 3$. For this purpose we only choose the LDM case to plot. We see that the $\mathrm{SZ}_{\mathrm{DM}}$ effect decreases with increasing $D_{0}$ or decreasing $\delta$. But when the diffusion parameters vary within a reasonable range, the basic conclusions remain unchanged.

In the above we have discussed various kinds of effects on $\mathrm{SZ}_{\mathrm{DM}}$, taking Draco for example. For other luminous dSphs as listed in Table 1, we present the values of $\Delta T$ at $35 \mathrm{GHz}$ and $1 \mathrm{THz}$ in Table 2 for the neutralino case, and in Table 3 for the LDM case. M99 profile is assumed. The particle physics parameters of DM are the same as described in Sec. 2.3. Since we have essentially assumed the same density profile for these dSphs, the magnitudes of their $\mathrm{SZ}_{\mathrm{DM}}$ effects are determined mostly by the the mass $\mathrm{M}_{\mathrm{vir}}$ of each galaxy. The differences in distance, on the other hand, do not affect the $\mathrm{SZ}_{\mathrm{DM}}$ effect. Thus, 


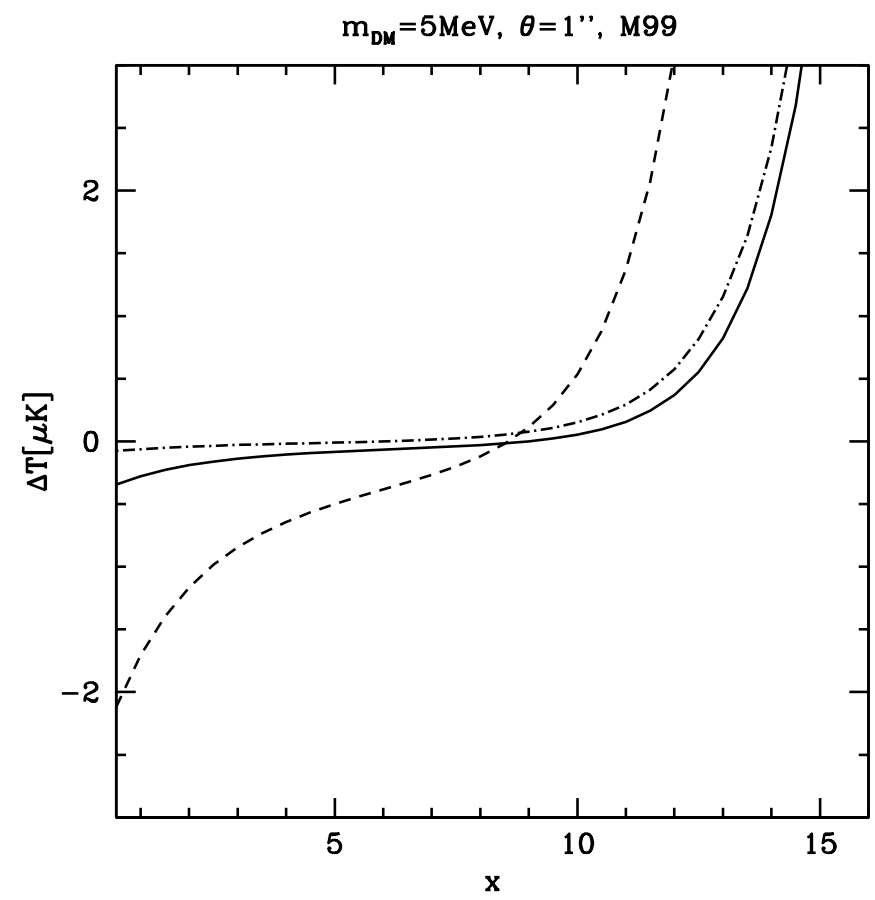

Figure 9: The temperature variation for beam size $1^{\prime \prime}$ induced by LDM annihilating in dark halo with M99 density profile. The solid line corresponds to our benchmark value $D_{0}=3.1 \times 10^{26} \mathrm{~cm}^{2} \mathrm{~s}^{-1}$ and $\delta=1 / 3$. The dot-dashed line is for the same $\delta$ but $D_{0}=1.0 \times 10^{27} \mathrm{~cm}^{2} \mathrm{~s}^{-1}$, and the dashed line is for the same $D_{0}$ but $\delta=2 / 3$. .

with these assumptions, $\mathrm{dSphs}$ with larger $\mathrm{M}_{\mathrm{vir}}$ would generally induce larger temperature distortion. This can be easily seen by comparing the result for Draco and Leo I, which have the same $r_{h}$ but different $\mathrm{M}_{\mathrm{vir}}$. Another essential factor in determining the final temperature distortion is the size of the diffusion zone in each dwarf galaxy. The value of $r_{h}$ is generally adopted as twice of the radius of the stellar component (if this value is larger than the tidal radius, we set $r_{h}=r_{t}$ ). For smaller $r_{h}$, a larger fraction of $e^{ \pm}$would escape away, and the $\mathrm{SZ}_{\mathrm{DM}}$ effect is also smaller. This is reflected clearly through the comparison between Fornax and Leo I.

Recently, the PAMELA [44, ATIC [45] and FERMI [46] observations of the cosmic ray electron and positron energy spectrum indicate a possible excess or even peak in the cosmic ray electrons and positrons. If this excess is due to DM annihilations, then the annihilation cross section must be "boosted" by a large factor compared with the usual expectation. Such a boost could be due to, e.g., "Sommerfeld enhancement" 47, 48, 49, 50, 51. However, in such a case the mass of DM particle must be of TeV scale, so even with a 1000-fold enhancement, the induced $\mathrm{SZ}_{\mathrm{DM}}$ effect, according to our estimate, would still be quite small. The DM substructures inside the dark halo are also thought to be able to "boost" the annihilation signal to some extent [52, 53]. However, the substructures near the center of the halo will be destroyed by tidal force. The "boost" is more effective at large radii [54]. Furthermore, for the cuspy profiles the central density is high enough to dominate the contribution over that from substructures. Hence the "boost", regardless of its origin, 
Table 2: Neutralino-like DM induced SZ effect (in units of K) for Local Group luminous dSphs. We assume $m_{\chi}=100 \mathrm{GeV},\langle\sigma v\rangle=3.0 \times 10^{-26} \mathrm{~cm}^{3} \mathrm{~s}^{-1}$, M99 profile, and $\theta=1^{\prime \prime}$.

\begin{tabular}{lcccc}
\hline \hline dSph & $r_{h}(\mathrm{kpc})$ & $M_{\text {vir }}\left(M_{\odot}\right)$ & $35 \mathrm{GHz}(x=0.616)$ & $1000 \mathrm{GHz}(x=17.46)$ \\
\hline Ursa Minor $^{\prime}$ & 1.6 & $4 \times 10^{9}$ & $-5.44 \times 10^{-12}$ & $2.43 \times 10^{-10}$ \\
Draco $^{a}$ & 1.6 & $4 \times 10^{9}$ & $-5.39 \times 10^{-12}$ & $2.32 \times 10^{-10}$ \\
Leo I & 1.6 & $1 \times 10^{9}$ & $-6.88 \times 10^{-13}$ & $5.37 \times 10^{-12}$ \\
Fornax & 5.4 & $1 \times 10^{9}$ & $-5.12 \times 10^{-13}$ & $1.91 \times 10^{-10}$ \\
Leo II & 1.04 & $4 \times 10^{8}$ & $-5.45 \times 10^{-15}$ & $3.35 \times 10^{-11}$ \\
Carina & 1.7 & $2 \times 10^{8}$ & $-2.37 \times 10^{-13}$ & $8.97 \times 10^{-13}$ \\
Sculptor & 3.26 & $1 \times 10^{9}$ & $-2.61 \times 10^{-12}$ & $9.72 \times 10^{-11}$ \\
Sextans $^{b}$ & 4.8 & $3 \times 10^{8}$ & $-1.61 \times 10^{-12}$ & $5.89 \times 10^{-11}$ \\
\hline \hline
\end{tabular}

${ }^{a} r_{\text {stellar }}$ is around $0.93 \mathrm{kpc}$, which give a slightly large $r_{h}$ as $1.86 \mathrm{kpc}$. In the calculation, we just use the $r_{h}=1.6 \mathrm{kpc}$ as the diffusion zone for consistency with previous results. ${ }^{b} r_{\text {stealer }}$ is around $4 \mathrm{kpc}$, which give a really large $r_{h}$ as $8 \mathrm{kpc}$. In the real calculation, we use $r_{t}$ as the diffusion zone.

Table 3: LDM induced SZ effect (in units of K) for Local Group luminous dSphs. We assume $m_{\mathrm{LDM}}=5 \mathrm{MeV},\langle\sigma v\rangle=2.5 \times 10^{-29} \mathrm{~cm}^{3} \mathrm{~s}^{-1}$, M99 profile, and $\theta=1^{\prime \prime}$.

\begin{tabular}{lcccc}
\hline \hline dSph & $r_{h}(\mathrm{kpc})$ & $M_{\text {vir }}\left(M_{\odot}\right)$ & $35 \mathrm{GHz}(x=0.616)$ & $1000 \mathrm{GHz}(x=17.46)$ \\
\hline Ursa Minor & 1.6 & $4 \times 10^{9}$ & $-3.11 \times 10^{-7}$ & $2.68 \times 10^{-5}$ \\
Draco & 1.6 & $4 \times 10^{9}$ & $-3.11 \times 10^{-7}$ & $2.67 \times 10^{-5}$ \\
Leo I & 1.6 & $1 \times 10^{9}$ & $-6.76 \times 10^{-8}$ & $1.48 \times 10^{-6}$ \\
Fornax & 5.4 & $1 \times 10^{9}$ & $-2.37 \times 10^{-7}$ & $3.80 \times 10^{-5}$ \\
Leo II & 1.04 & $4 \times 10^{8}$ & $-1.63 \times 10^{-8}$ & $4.76 \times 10^{-6}$ \\
Carina & 1.7 & $2 \times 10^{8}$ & $-1.97 \times 10^{-8}$ & $1.71 \times 10^{-6}$ \\
Sculptor & 3.26 & $1 \times 10^{9}$ & $-1.37 \times 10^{-7}$ & $1.48 \times 10^{-5}$ \\
Sextans & 4.8 & $3 \times 10^{8}$ & $-7.76 \times 10^{-8}$ & $1.09 \times 10^{-5}$ \\
\hline \hline
\end{tabular}

would have little impact on our present calculation.

\section{Conclusion}

In this paper, we calculate the non-thermal SZ effect induced by the energetic electrons and positrons produced by the annihilation of neutralino and LDM in dSphs. We take the Draco dwarf as an example to present most of our results, but we have also applied the same calculation to other luminous dSphs in the Local Group.

In our calculation, we obtain the equilibrium $e^{ \pm}$distribution by fully solving the diffusion equation with the Green's function method. We take synchrotron emission, ICS, ionization loss as well as diffusion loss of $e^{ \pm}$into account. We find that for small scale systems such as the dwarf galaxies, diffusion effect is crucial. The $e^{ \pm}$distribution is much less steep than the source function which is proportional to $\rho_{\mathrm{DM}}^{2}$. This important effect 
was mentioned in [1] but was neglected in the numerical calculations in their previous analysis [16], and the resulting $e^{ \pm}$density could be different by several orders of magnitude. Another difference is that we considered contributions from $e^{ \pm}$with relatively low energy (i.e. we do not use any arbitrary energy cut off). Such low energy $e^{ \pm}$could still contribute significantly to the $\mathrm{SZ}_{\mathrm{DM}}$ signal.

The SZ effect by the non-thermal $e^{ \pm}$depends on the density profile of the DM halo. We considered M99, NFW and B95 profiles. As expected, for the cuspy M99 profile the effect is much more significant. Nevertheless, for all profiles we find much smaller signals than previously claimed. Due to the diffusion effect, even for the strongly cuspy M99 profile, the SZ effect predicted for neutralino is too small to be observed with the current or coming generation of instruments. The possible astrophysical foregrounds or contamination may even make the detection more difficult.

Considering that a smaller mass of DM will result in a higher DM number density and annihilation rate, we also investigated the case of LDM, i.e. DM candidate with $\mathrm{MeV}$ mass scale. In this case, there is some hope of detecting the DM induced SZ effect, though the effect is also much smaller than previous claims. For the strongly cusped profile assumed, $\Delta T$ could reach tens of $\mu \mathrm{K}$ when the frequency is around or larger than THz. However, we note that $\mathrm{THz}$ observation is difficult to do on ground, with the possible exception of a few sites in the Antarctica high plateau.

One of the largest uncertainty in our calculation is the diffusion coefficient of the dSphs, which depends on the magnetic field strength, for which we have no data. We made an assumption on its form and value for the present calculation. It is possible that the magnetic field is much weaker than we assumed, in that case, the SZ effect by non-thermal $e^{ \pm}$would be even smaller.

These results show that the non-thermal SZ effect induced by DM annihilation in dSphs is small and difficult to observe, even though the expected astrophysical contamination is small. Combined with our earlier results for clusters (Paper I), we conclude that the non-thermal $\mathrm{SZ}_{\mathrm{DM}}$ effect is perhaps not a powerful method to detect or constrain DM annihilations.

\section{Acknowledgments}

We thank Pengjie Zhang, Bin Yue, and the anonymous referee for helpful discussion and suggestions. This work is supported by the National Science Foundation of China under grants 10773011, 10525314, 10533010, by the Chinese Academy of Sciences under grant No. KJCX3-SYW-N2, and by the Ministry of Science and Technology National Basic Science Program (Project 973) under grants 2007CB815401 and 2010CB833000, and by the National Basic Research Program of China under Grant No. 2009CB824800.

\section{References}

[1] S. Colafrancesco, S. Profumo, and P. Ullio, Detecting dark matter WIMPs in the Draco dwarf: A multiwavelength perspective, Phys. Rev. D $\mathbf{7 5}$ (Jan., 2007) 023513-+, arXiv:astro-ph/0607073. 
[2] G. Jungman, M. Kamionkowski, and K. Griest, Supersymmetric dark matter, Phys. Rept. 267 (Mar., 1996) 195-373, arXiv:hep-ph/9506380.

[3] C. Bœhm and P. Fayet, Scalar dark matter candidates, Nuclear Physics B 683 (Apr., 2004) 219-263, arXiv:hep-ph/0305261.

[4] Y. B. Zeldovich, A. A. Klypin, M. Y. Khlopov, and V. M. Chechetkin, Astrophysical constraints on the mass of heavy stable neutral leptons, Sov. J. Nucl. Phys. 31 (1980) 664-669.

[5] S. Rudaz and F. W. Stecker, cosmic ray anti-protons, positrons and gamma-rays from halo dark matter annihilation, Astrophys. J. 325 (1988) 16.

[6] J. R. Ellis et al., cosmic ray constraints on the annihilation of relic particles in the galactic halo, Phys. Lett. B214 (1988) 403.

[7] M. Kamionkowski and M. S. Turner, A Distinctive positron feature from heavy WIMP annihilations in the galactic halo, Phys. Rev. D43 (1991) 1774-1780.

[8] D. Fargion, M. Y. Khlopov, R. V. Konoplich, and R. Mignani, Bounds on very heavy relic neutrinos by their annihilation in galactic halo, Phys. Rev. D52 (1995) 1828-1836.

[9] R. A. Sunyaev and Y. B. Zeldovich, Small-Scale Fluctuations of Relic Radiation, Astrophys. Space Sci. 7 (Apr., 1970) 3-19.

[10] R. A. Sunyaev and Y. B. Zeldovich, The interaction of matter and radiation in the hot model of the Universe, II, Astrophys. Space Sci. 7 (Apr., 1970) 20-30.

[11] S. Colafrancesco, P. Marchegiani, and E. Palladino, The non-thermal Sunyaev-Zel'dovich effect in clusters of galaxies, Astron. Astrophys. 397 (Jan., 2003) 27-52, arXiv:astro-ph/0211649.

[12] T. A. Enßlin and C. R. Kaiser, Comptonization of the cosmic microwave background by relativistic plasma, Astron. Astrophys. 360 (Aug., 2000) 417-430, arXiv:astro-ph/0001429.

[13] S. Colafrancesco, S. Profumo, and P. Ullio, Multi-frequency analysis of neutralino dark matter annihilations in the Coma cluster, Astron. Astrophys. 455 (Aug., 2006) 21-43, arXiv: astro-ph/0507575.

[14] S. Colafrancesco, SZ effect from Dark Matter annihilation, Astron. Astrophys. 422 (July, 2004) L23-L27, arXiv:astro-ph/0405456.

[15] S. Colafrancesco, P. de Bernardis, S. Masi, G. Polenta, and P. Ullio, Direct probes of dark matter in the cluster 1ES0657-556 through microwave observations, Astron. Astrophys. 467 (May, 2007) L1-L5, arXiv:astro-ph/0702568].

[16] T. L. Culverhouse, N. W. Evans, and S. Colafrancesco, Comptonization of cosmic microwave background photons in dwarf spheroidal galaxies, Mon. Not. Roy. Astron. Soc. 368 (May, 2006) 659-667, arXiv: astro-ph/0602093.

[17] Q. Yuan, X. Bi, F. Huang, and X. Chen, Dark matter annihilation and non-thermal SunyaevZel'dovich effect: I. galaxy cluster, JCAP 0910 (2009) 013, arXiv:0902.4294].

[18] J. Lavalle, C. Bœehm, and J. Barthès, On the Sunyaev-Zel'dovich effect from dark matter annihilation or decay in galaxy clusters, Journal of Cosmology and Astro-Particle Physics 2 (Feb., 2010) 5-+, arXiv:0907.5589. 
[19] A. Achterberg, R. Blandford, and V. Periwal, Two-fluid models of cosmic ray shock acceleration, Astron. Astrophys. 132 (Mar., 1984) 97-104.

[20] E. Nakar, M. Milosavljević, and D. Nagai, Cluster Merger Shock Constraints on Particle Acceleration and Nonthermal Pressure in the Intracluster Medium, Astrophys. J. 675 (Mar., 2008) 126-135, arXiv:0706.2881.

[21] A. Burkert, The Structure of Dark Matter Halos in Dwarf Galaxies, Astrophys. J. Lett. 447 (July, 1995) L25, arXiv:astro-ph/9504041.

[22] J. F. Navarro, C. S. Frenk, and S. D. M. White, A Universal Density Profile from Hierarchical Clustering, Astrophys. J. 490 (1997) 493-508, astro-ph/9611107.

[23] B. Moore, T. R. Quinn, F. Governato, J. Stadel, and G. Lake, Cold collapse and the core catastrophe, Mon. Not. Roy. Astron. Soc. 310 (1999) 1147-1152, astro-ph/9903164.

[24] V. S. Berezinsky, A. V. Gurevich, and K. P. Zybin, Distribution of dark matter in the Galaxy and the lower limits for the masses of supersymmetric particles., Physics Letters B 294 (Nov., 1992) 221-228.

[25] G. L. Bryan and M. L. Norman, Statistical Properties of X-Ray Clusters: Analytic and Numerical Comparisons, Astrophys. J. 495 (Mar., 1998) 80, arXiv:astro-ph/9710107.

[26] J. S. Bullock, T. S. Kolatt, Y. Sigad, R. S. Somerville, A. V. Kravtsov, A. A. Klypin, J. R. Primack, and A. Dekel, Profiles of dark haloes: evolution, scatter and environment, Mon. Not. Roy. Astron. Soc. 321 (Mar., 2001) 559-575, arXiv:astro-ph/9908159].

[27] K. Dolag, M. Bartelmann, F. Perrotta, C. Baccigalupi, L. Moscardini, M. Meneghetti, and G. Tormen, Numerical study of halo concentrations in dark-energy cosmologies, Astron. Astrophys. 416 (Mar., 2004) 853-864, arXiv:astro-ph/0309771.

[28] N. W. Evans, F. Ferrer, and S. Sarkar, A 'Baedecker' for the dark matter annihilation signal, Phys. Rev. D69 (2004) 123501, astro-ph/0311145.

[29] N. W. Evans, The power-law galaxies, Mon. Not. Roy. Astron. Soc. 267 (Mar., 1994) $333-360$.

[30] N. W. Evans, F. Ferrer, and S. Sarkar, A 'Baedecker' for the dark matter annihilation signal, Phys. Rev. D69 (2004) 123501, astro-ph/0311145.

[31] M. G. Walker, M. Mateo, E. W. Olszewski, O. Y. Gnedin, X. Wang, B. Sen, and M. Woodroofe, Velocity Dispersion Profiles of Seven Dwarf Spheroidal Galaxies, Astrophys. J. Lett. 667 (Sept., 2007) L53-L56, arXiv:0708.0010.

[32] P. Gondolo et al., DarkSUSY: Computing supersymmetric dark matter properties numerically, JCAP 0407 (2004) 008, astro-ph/0406204].

[33] P. Jean, J. Knödlseder, V. Lonjou, M. Allain, J.-P. Roques, G. K. Skinner, B. J. Teegarden, G. Vedrenne, P. von Ballmoos, B. Cordier, P. Caraveo, R. Diehl, P. Durouchoux, P. Mandrou, J. Matteson, N. Gehrels, V. Schönfelder, A. W. Strong, P. Ubertini, G. Weidenspointner, and C. Winkler, Early SPI/INTEGRAL measurements of $511 \mathrm{keV}$ line emission from the 4th quadrant of the Galaxy, Astron. Astrophys. 407 (Aug., 2003) L55-L58, arXiv:astro-ph/0309484. 
[34] P. Jean, J. Knödlseder, V. Lonjou, M. Allain, J.-P. Roques, G. K. Skinner, B. J. Teegarden, G. Vedrenne, P. von Ballmoos, B. Cordier, P. Caraveo, R. Diehl, P. Durouchoux, P. Mandrou, J. Matteson, N. Gehrels, V. Schönfelder, A. W. Strong, P. Ubertini, G. Weidenspointner, and C. Winkler, Early SPI/INTEGRAL measurements of $511 \mathrm{keV}$ line emission from the 4th quadrant of the Galaxy, Astron. Astrophys. 407 (Aug., 2003) L55-L58, arXiv:astro-ph/0309484.

[35] C. Boehm, D. Hooper, J. Silk, M. Casse, and J. Paul, MeV dark matter: Has it been detected?, Phys. Rev. Lett. 92 (2004) 101301, astro-ph/0309686.

[36] J. F. Beacom, N. F. Bell, and G. Bertone, Gamma-ray constraint on Galactic positron production by MeV dark matter, Phys. Rev. Lett. 94 (2005) 171301, astro-ph/0409403.

[37] Y. Ascasibar, P. Jean, C. Boehm, and J. Knoedlseder, Constraints on dark matter and the shape of the Milky Way dark halo from the $511 \mathrm{keV}$ line, Mon. Not. Roy. Astron. Soc. $\mathbf{3 6 8}$ (2006) 1695-1705, astro-ph/0507142.

[38] J. F. Beacom and H. Yuksel, Stringent Constraint on Galactic Positron Production, Phys. Rev. Lett. 97 (2006) 071102, astro-ph/0512411.

[39] P. Sizun, M. Casse, and S. Schanne, Continuum gamma-ray emission from light dark matter positrons and electrons, Phys. Rev. D74 (2006) 063514, astro-ph/0607374.

[40] L. Zhang, X.-L. Chen, Y.-A. Lei, and Z.-G. Si, The impacts of dark matter particle annihilation on recombination and the anisotropies of the cosmic microwave background, Phys. Rev. D74 (2006) 103519, astro-ph/0603425.

[41] G. Weidenspointner et al., An asymmetric distribution of positrons in the Galactic disk revealed by $\gamma$-rays, Nature 451 (2008) 159-162.

[42] S. Longair, high energy astrophysics. cambridge university press, 1992.

[43] T. E. Jeltema and S. Profumo, Searching for Dark Matter with X-ray Observations of Local Dwarf Galaxies, arXiv:0805.1054.

[44] PAMELA Collaboration, O. Adriani et al., An anomalous positron abundance in cosmic rays with energies 1.5.100 GeV, Nature 458 (2009) 607-609, arXiv:0810.4995.

[45] J. Chang, J. H. Adams, H. S. Ahn, G. L. Bashindzhagyan, M. Christl, O. Ganel, T. G. Guzik, J. Isbert, K. C. Kim, E. N. Kuznetsov, M. I. Panasyuk, A. D. Panov, W. K. H. Schmidt, E. S. Seo, N. V. Sokolskaya, J. W. Watts, J. P. Wefel, J. Wu, and V. I. Zatsepin, An excess of cosmic ray electrons at energies of 300-800GeV, Nature 456 (Nov., 2008) 362-365.

[46] The Fermi LAT Collaboration, A. A. Abdo et al., Measurement of the Cosmic Ray e+ plus e- spectrum from $20 \mathrm{GeV}$ to $1 \mathrm{TeV}$ with the Fermi Large Area Telescope, Phys. Rev. Lett. 102 (2009) 181101, arXiv:0905.0025.

[47] M. Cirelli, M. Kadastik, M. Raidal, and A. Strumia, Model-independent implications of the e+/e-, pbar cosmic ray spectra on properties of Dark Matter, Nuclear Physics B $\mathbf{8 1 3}$ (May, 2009) 1-2, arXiv:0809.2409.

[48] N. Arkani-Hamed, D. P. Finkbeiner, T. R. Slatyer, and N. Weiner, A theory of dark matter, Phys. Rev. D 79 (Jan., 2009) 015014, arXiv:0810.0713.

[49] M. Pospelov and A. Ritz, Astrophysical signatures of secluded dark matter, Physics Letters B 671 (Jan., 2009) 391-397, arXiv:0810.1502. 
[50] J. March-Russell, S. M. West, D. Cumberbatch, and D. Hooper, Heavy dark matter through the Higgs portal, Journal of High Energy Physics 7 (July, 2008) 58, arXiv:0801.3440.

[51] J. March-Russell and S. M. West, WIMPonium and Boost Factors for Indirect Dark Matter Detection, ArXiv e-prints (Dec., 2008) arXiv:0812.0559.

[52] Q. Yuan and X. J. Bi, The galactic positron flux and dark matter substructures, Journal of Cosmology and Astro-Particle Physics 5 (May, 2007) 1, arXiv:astro-ph/0611872.

[53] J. Lavalle, Q. Yuan, D. Maurin, and X. Bi, Full calculation of clumpiness boost factors for antimatter cosmic rays in the light of $\Lambda C D M N$-body simulation results. Abandoning hope in clumpiness enhancement?, Astron. Astrophys. 479 (Feb., 2008) 427-452, arXiv:0709.3634.

[54] X. J. Bi, Gamma rays from the neutralino dark matter annihilations in the Milky Way substructures, Nuclear Physics B $\mathbf{7 4 1}$ (May, 2006) 83-107, [arXiv:astro-ph/0510714]. 


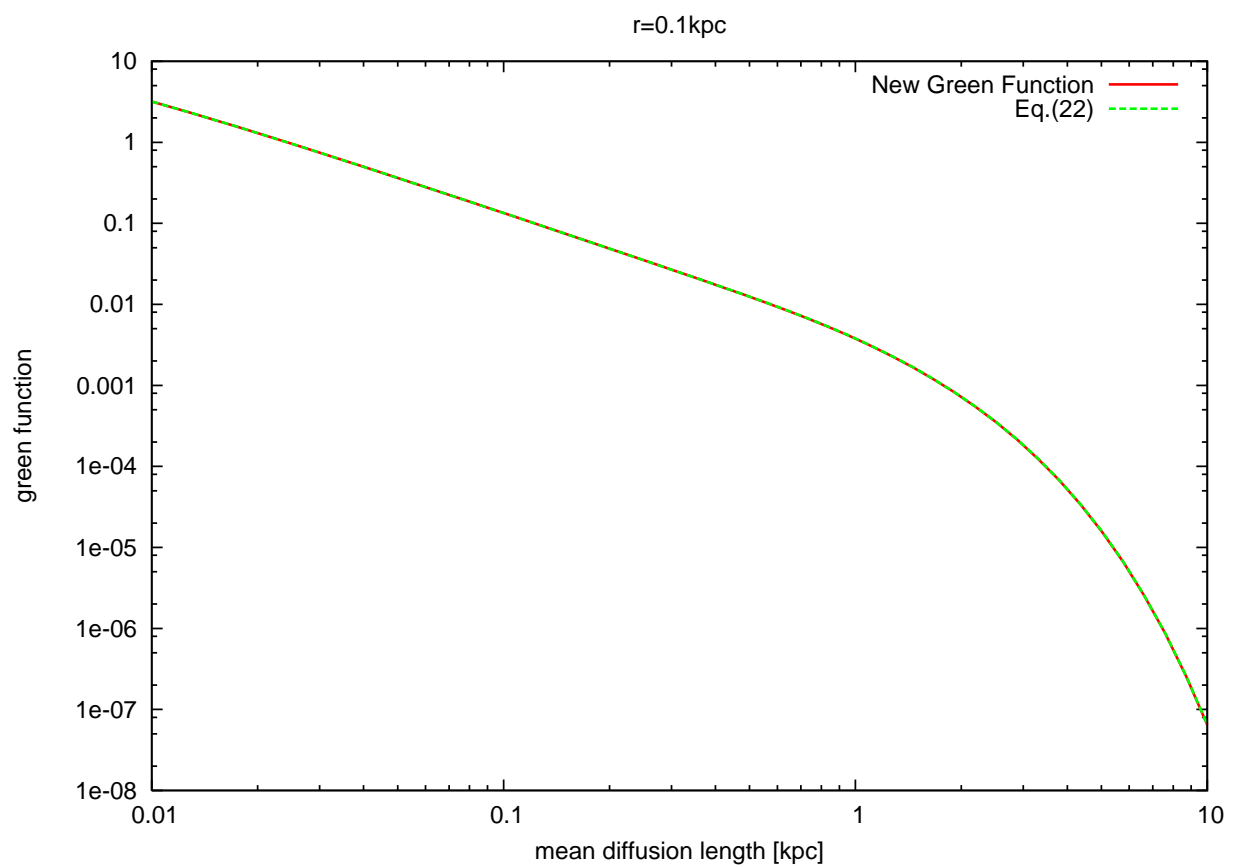




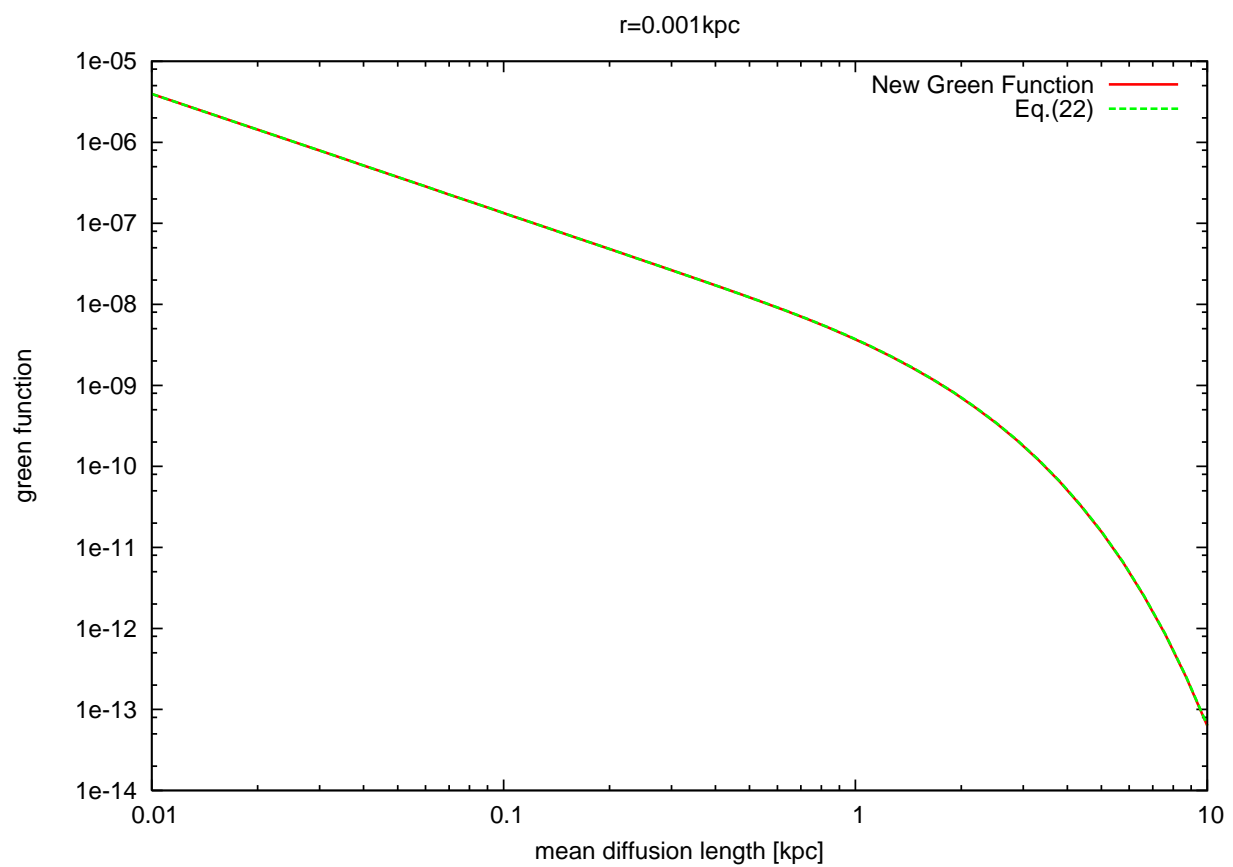




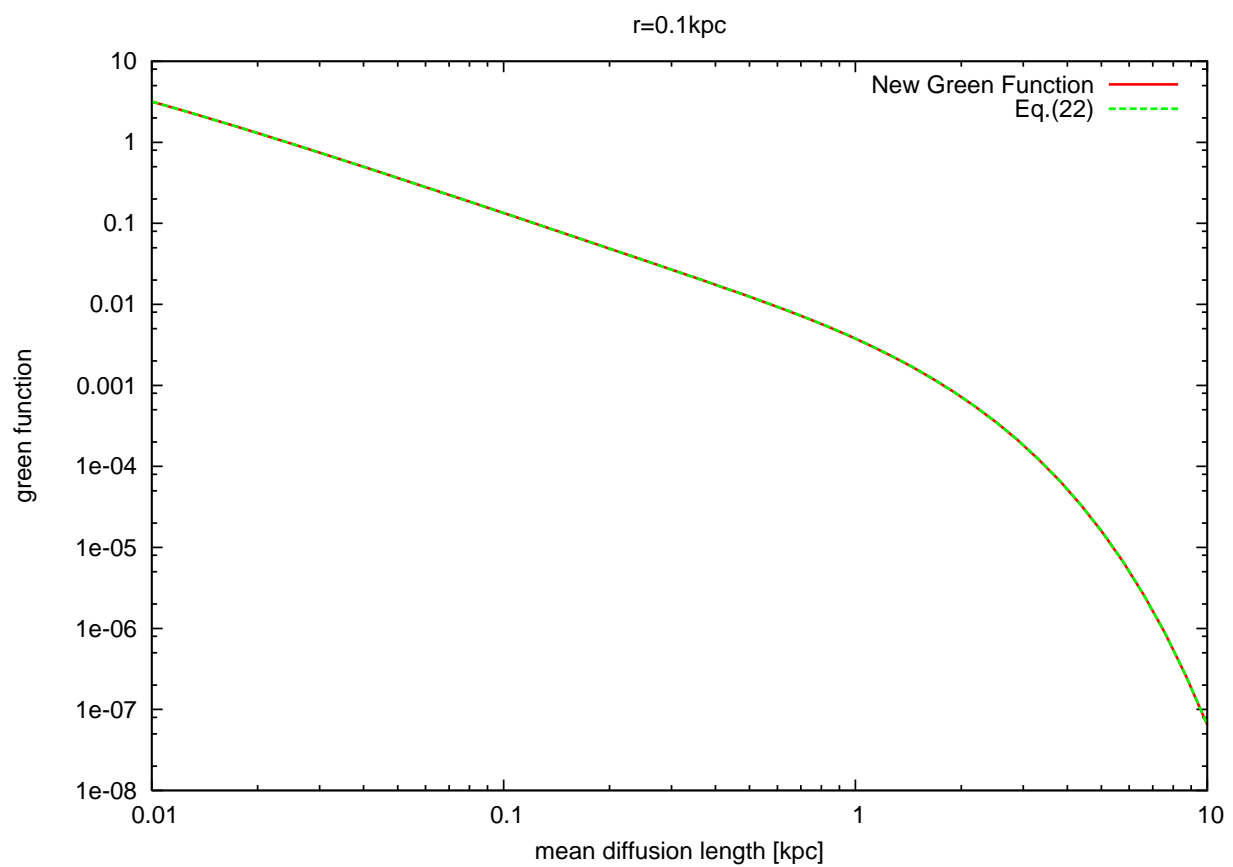




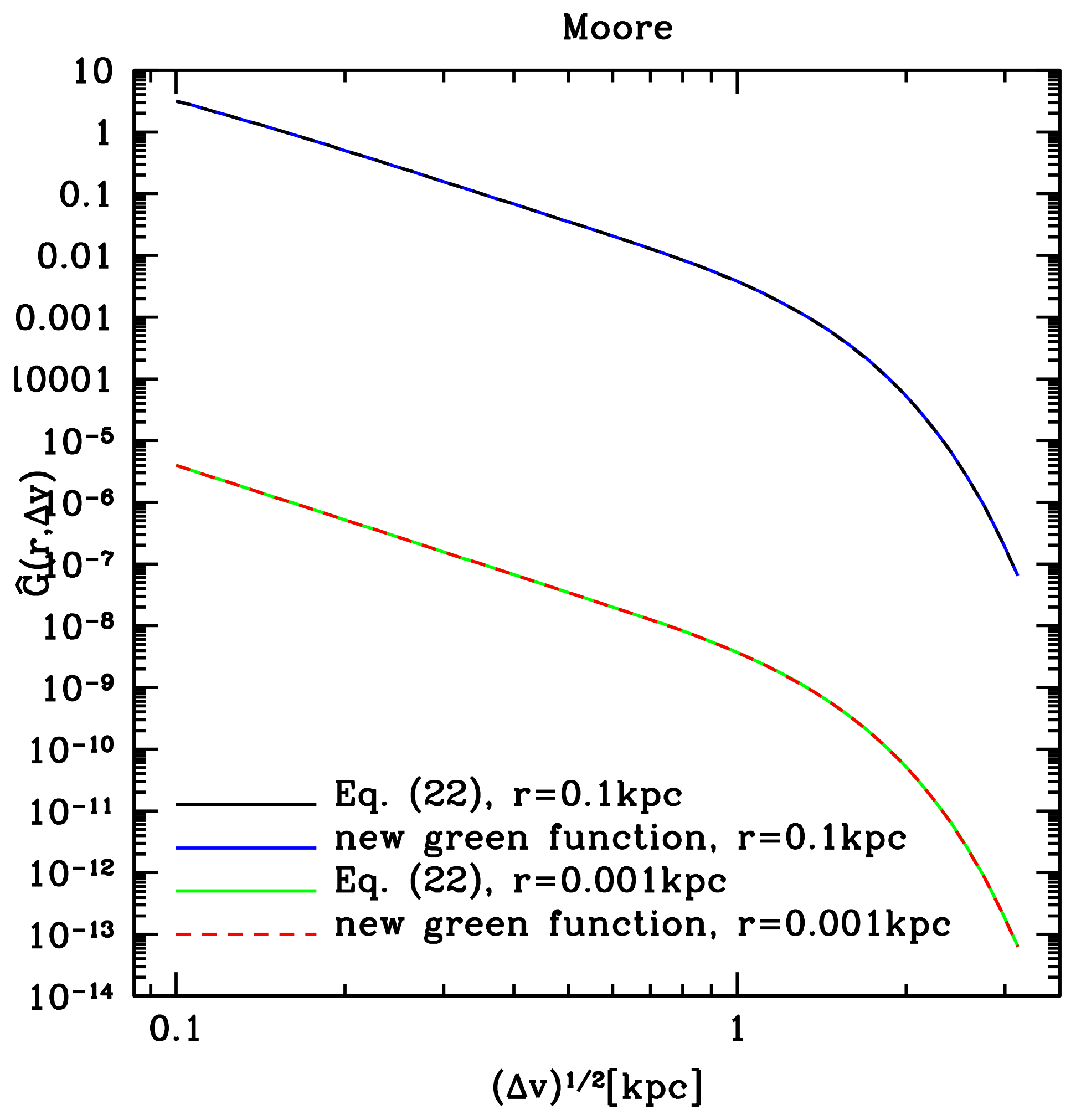

